DOI: 10.20472/IAC.2018.036.006

\title{
PAITOON CHETTHAMRONGCHAI
}

Kasetsart University, Thailand

\section{CONSUMER BEHAVIOR AND CUSTOMER LOYALTY PROGRAM: CASE OF TESCO IN THAILAND}

\begin{abstract}
:
Loyalty programs (sometimes referred to as loyalty systems) are part of marketing communication, respectively one of the classic tools of sales promotion. Through this tool marketing can effect on customers in order to stimulate their loyalty. The basic principle of loyalty program is to motivate customers to more frequent purchases or multiple purchases whether of goods or services. The essence of this tool is to reward consumers for their behavior. The primary purpose of loyalty programs is to establish and maintain a loyal customer long term relationship (Zamazalova, 2010). If customers are involved in loyalty programs they can acquire various forms of rewards for their purchases. Various kinds of rewards (discounts, gifts or bonuses ...) are key factors on which the loyalty program is built and which is the basic of the motivation. Loyalty marketing is based on the fact that $20 \%$ of customers at the company can create up to $80 \%$ of sales. Moreover the most loyal customers are most profitable for the company (O'Brien and Jones, 1995). Loyalty programs are regarded as a tool through which you can build long term loyalty. The essence of a functioning loyalty program is to provide rewards to customers who often buy in a certain.

The paper introduces the partial results of the marketing research on Tesco loyalty programs in Thailand. The aim was to identify the reasons that lead consumers to participate in loyalty programs and the benefits that are the main motivators for participation in the loyalty program.
\end{abstract}

\section{Keywords:}

Loyalty programs, marketing communication, loyal customers 


\section{Introduction}

Today's constantly changing and very competitive business environment is requiring sensitive awareness of the situation, and fast reaction abilities from the companies in order to provide products and services to meet customers' expectations. Long and reliable customers' relationships are valued by the companies, and customers are expecting to receive different benefits when shopping on a regular basis in some certain store or retail chains. Loyalty programs have a remarkable impact to the business. Loyalty programs can also be considered to be a business strategy for the company, and can be executed effectively via well-organized business processes, which suite best for company's communication with the customers.

Loyalty programs are frequently referred to as "points" or "rewards" programs. They are offered by airlines, grocery stores, gas stations, hotels, car rental agencies, restaurants, coffee shops, book stores and so on (Baran, Galka and Strunk, 2008). The success of the program lies in providing customers with specific rewards. The intention is to reward loyal, frequent buyers more often. The distinction is made between good and bad customers with the aid of behavior registration (Peelen, 2005). As articulated by Reichheld and Saaser (1990) and Reichheld and Teal (1996), loyal customers enable firms to generate more profit over time because of various reasons; increased purchases, reduced operating costs and increased profit margin. As competition intensified during the current economic crisis, many firms are developing or improving their loyalty programs to deter customers from defecting to their competitors.

\section{Literature Review}

\subsection{Customer Loyalty}

Loyalty as a concept has existed since the beginning of the 20th century, and was merely observed as a term for repetitive purchase behavior. It was not until Jacoby and Chestnut (1978) studied the drivers of loyalty that it became a multifaceted theoretical concept. Today many definitions and variations of loyalty exist, but in general researchers agrees that loyalty can be viewed as either of following: behavioral loyalty, or attitudinal loyalty. Customer's loyalty is a customer's attachment to a brand, store, manufacturer, service provider or other entities based on favorable attitudes and behavioral responses such as repeat purchase (Baran, Galka and Strunk, 2008). Customer loyalty is very essential to the organization in order to retain its current customers. It is because customer's loyalty can serve several benefits to the organization. Loyal customers are less price-sensitive, reduce marketing expenditures for attracting new customers and improved organizational profitability (Rowley, 2005). Raman (1999) and Bowen and Chen (2001) stated that, loyal customers serve as a "fantastic marketing force" by providing recommendations and 
spreading positive word of mouth. Moreover, loyal customers cost less to serve, in part because they know the products and require less information (Bowen and Chen, 2001).

\subsection{Loyalty Program}

Broekhuizen and Peelen, (1997) siad that loyalty programs are designed to improve customer's satisfaction and commitment. There are many divisions of loyalty programmes. In this paper, only such divisions that have been taken into account in the pursuance of content analysis are introduced. The basic of loyalty programmes is concerned with distinguishing the target group of any one particular loyalty programme, so, there are programmes for end customers and programmes for retailers (Mrkosová et al., 2011). The next loyalty programmes is orientated to the point of view of time. Bellini et al. (2011) mention long and short term loyalty building activities. Short term activities include mini collections and special promotions. According to Janouch (2010, p. 201), short term activities are, merely, a form of sales promotion. He suggests that a loyalty programme should be available to a customer for a long time and this does not correspond to the principle of short term programmes. The effectiveness and the relevancy of loyalty program are measured through the achievement of certain levels of customer's satisfaction threshold (Keh and Lee, 2006). Loyalty programs give away benefits or the perceived value to the members as a token of appreciation for their loyalty to the organization. In addition, Bolton, Kannan, and Bramlett (2000) stated that when customers involve in the loyalty programs, the perceived benefits they receive will lead to their loyalty. For that reason, the benefits of customer loyalty program can be divided into two; hard and soft benefits. The hard benefits are the monetary benefits in the forms of rebates, special discounts and coupons meanwhile the soft benefits are the non-monetary benefits (Mulhern and Duffy, 2004).

\subsection{Loyalty Programs in Grocery Retailing}

Many retailers employ some form of frequency reward programs (loyalty programs) striving to find ways in which to attract and retain their customers. It is typically expected from these programs that a rewarded customer will repeat his or her purchase. Although many retailers have introduced these programs, we do not have sufficient information about how these different ways of rewarding influence consumer behavior. In grocery retailing, there are numerous competitors, and individual retailers do not have many opportunities to show how they are different. Airline companies are exposed to a similar situation. Their services are perceived as all being similar to each other (i.e. to be no different from one another). Loyalty programs have been successfully proven by many airline companies and this was the inspiration for grocery retailers (Lal and Bell, 2003). Retailers try to influence customer behavior by means of their loyalty programs; they want their customers to stay loyal to their company or their brand. On this basis, retailers want to build long term, profitable 
relationships with their customers (Zamazalová, 2009). Simply said, it is concerned with providing various rewards and incentives to customers for their repeated purchases (Lal and Bell, 2003). Vesel and Zabkar (2009) perceive loyalty programs as "a discriminative marketing tool" because companies reward only some of their customers (i.e. those who are members), not every buyer. It has emerged from recent research that the biggest interest in loyalty programs in the retailing sector is in the area of groceries (Ott, 2011). This information confirms the importance of research on the retailing sector.

\section{Research Methodology}

A quantitative and qualitative method have been used to achieve the objectives of this study, in which descriptive research design was applied as the framework of the study. Quantitative approach and sample survey method by questionnaire was applied for the study. The population is all customers who live in Bangkok and have shopping experience in term of buying product at Tesco Lotus since January 2015. The sample was selected by non-probability in convenience technique which was 385 respondents. The data was collected during 26 December 2015 - 25 January 2016.

Secondary data was gathered from theory, textbook, journal and thesis related to the study while primary data was collected through questionnaire via Google Forms and selfadministration. Customer Characteristics were interpreted by descriptive statistics including frequency and percentage. The hypotheses were tested by multiple regression by determining $95 \%$ of confidence level. The reliability of the survey was measured by Cronbach's Alpha which the acceptable for exploratory research was equal or greater than 0.70 .

\section{Part 1 : Customer Characteristics analysis}

Customer characteristics including gender, age, educational background, monthly income, current residence who normally shopped at Tesco Lotus were interpreted by descriptive statistics as shown in table 1. 
Table 1 Frequency and percent of customer characteristics

$$
(\mathrm{n}=385)
$$

\begin{tabular}{lccc}
\hline Customer Characteristics & Frequency & Percent & $\begin{array}{c}\text { Cumulative } \\
\text { Percent }\end{array}$ \\
\hline $\begin{array}{l}\text { 1. Gender } \\
\text { Male }\end{array}$ & 112 & 29.1 & 29.1 \\
$\quad$ Female & 273 & 70.9 & 100.0 \\
2. Age & 7 & 1.8 & 1.8 \\
$\quad$ Less than 20 years & 158 & 41.0 & 42.9 \\
20 - 30 years & 135 & 35.1 & 77.9 \\
31 - 40 years & 64 & 16.6 & 94.5 \\
$\quad$ 41 - 50 years & 21 & 5.5 & 100.0 \\
$\quad$ Above 50 years & & & \\
3. Educational background & & & \\
$\quad$ Lower Bachelor Degree & 35 & 9.1 & 9.1 \\
$\quad$ Bachelor Degree or & 211 & 54.8 & 63.9 \\
equivalent & 134 & 34.8 & 98.7 \\
$\quad$ Master Degree or & 5 & 1.3 & 100.0 \\
equivalent & & & \\
Doctor Degree or & & & \\
equivalent & 16 & 4.2 & 4.2 \\
4. Monthly Income & 73 & 19.0 & 23.1 \\
$\quad$ Less than 10,000 baht & 61 & 15.8 & 39.0 \\
10,001 - 20,000 baht & 66 & 17.1 & 56.1 \\
20,001 - 30,000 baht & 42 & 10.9 & 67.0 \\
30,001 - 40,000 baht & 127 & 33.0 & 100.0 \\
$\quad$ 40,001 - 50,000 baht & $\mathbf{1 0 0}$ & \\
$\quad$ Above 50,000 baht & & & \\
\hline Total &
\end{tabular}

According to table 1 , it illustrated that most respondents were female $(70.9 \%)$ and male $(29.1 \%)$ respectively. Their age were mostly in $20-30$ years $(41 \%)$ and 31 - 40 years (35.1\%) while their educational background were mainly in Bachelor Degree or equivalent (54.8\%) and Master Degree or equivalent (34.8\%). Monthly income above 50,000 baht (33\%) was shown as the highest frequency, followed by 10,001 - 20,000 baht (19\%). 


\section{Part 2 : Customer's attitude toward Key Success Factors of Store Loyalty}

Six constructs of key success factors were evaluated in each question by frequency, percent, mean, standard deviation and level of agreement.

Table 2 Frequency, percent, mean, standard deviation and level of agreement of customer's attitude toward key success factors of store loyalty.

$$
(\mathrm{n}=385)
$$

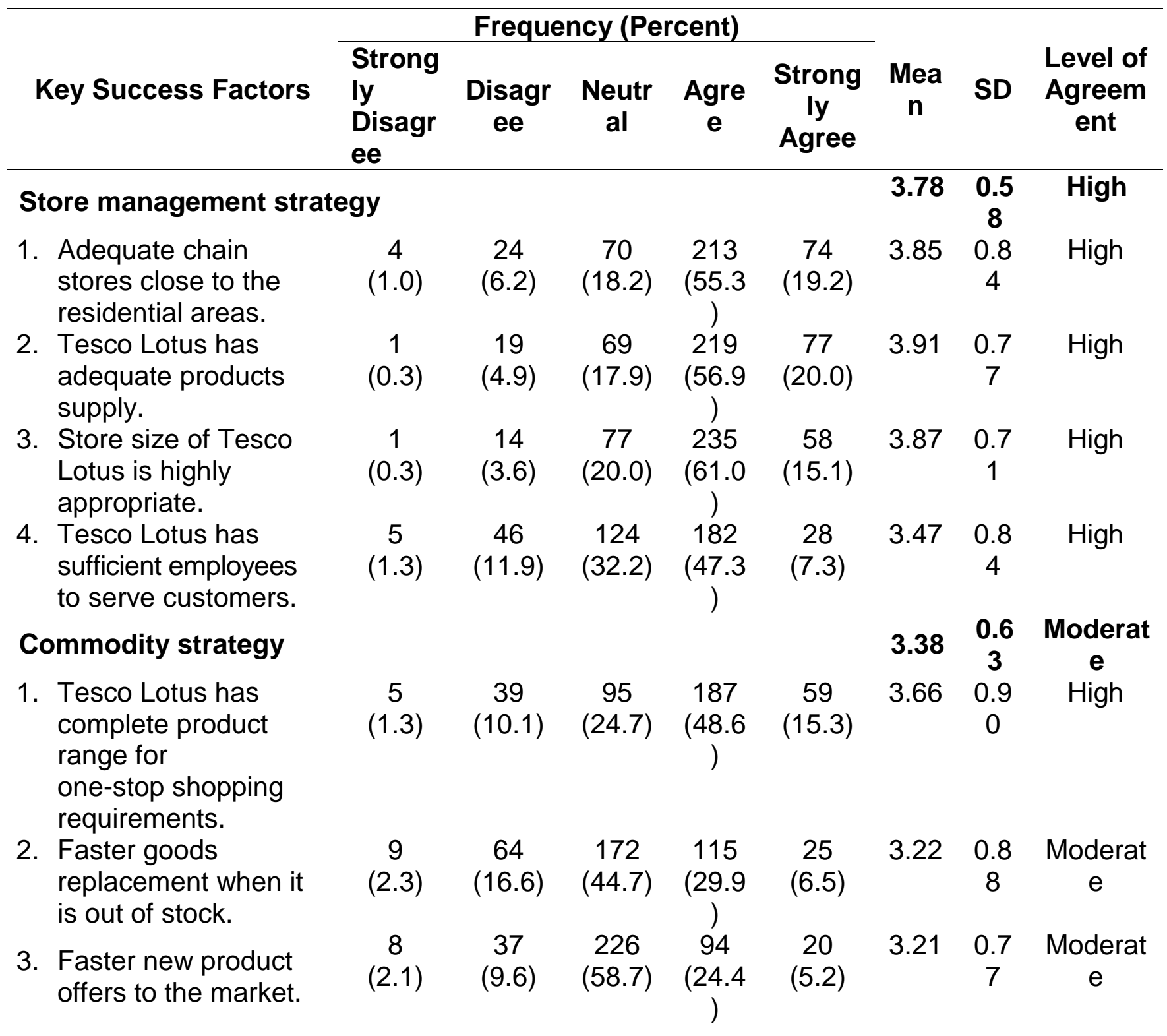


4. Unique commodities can be found in Tesco Lotus.

5. Neatness on goods display and layout. $\begin{array}{ccccc}9 & 59 & 202 & 92 & 23 \\ (2.3) & (15.3) & (52.5) & (23.9 & (6.0)\end{array}$

$\begin{array}{llll}4 & 22 & 120 & 203\end{array}$

(1.0)

$(5.7) \quad(31.2) \quad(52.7$
36

(9.4)
3.160 .8 Moderat

e

$3.64 \quad 0.7 \quad$ High

$$
(n=385)
$$

\begin{tabular}{|c|c|c|c|c|c|c|c|c|}
\hline \multirow[b]{2}{*}{ Key Success Factors } & \multicolumn{5}{|c|}{ Frequency (Percent) } & \multirow[b]{2}{*}{$\begin{array}{c}\text { Mea } \\
\mathbf{n}\end{array}$} & \multirow[b]{2}{*}{ SD } & \multirow[b]{2}{*}{$\begin{array}{l}\text { Level of } \\
\text { Agreem } \\
\text { ent }\end{array}$} \\
\hline & $\begin{array}{l}\text { Strong } \\
\text { ly } \\
\text { Disagr } \\
\text { ee }\end{array}$ & $\begin{array}{l}\text { Disagr } \\
\text { ee }\end{array}$ & $\begin{array}{l}\text { Neutr } \\
\text { al }\end{array}$ & $\begin{array}{l}\text { Agre } \\
\mathrm{e}\end{array}$ & $\begin{array}{l}\text { Strong } \\
\text { ly } \\
\text { Agree }\end{array}$ & & & \\
\hline \multicolumn{6}{|l|}{ Promotion strategy } & 3.81 & $\begin{array}{c}0.6 \\
7\end{array}$ & High \\
\hline $\begin{array}{l}\text { 1. Tesco Lotus offers } \\
\text { creative promotion } \\
\text { strategies. }\end{array}$ & $\begin{array}{c}8 \\
(2.1)\end{array}$ & $\begin{array}{c}14 \\
(3.6)\end{array}$ & $\begin{array}{c}125 \\
(32.5)\end{array}$ & $\begin{array}{c}180 \\
(46.8 \\
\quad)\end{array}$ & $\begin{array}{c}58 \\
(15.1)\end{array}$ & 3.69 & $\begin{array}{c}0.8 \\
5\end{array}$ & High \\
\hline $\begin{array}{l}\text { 2. Having promotional } \\
\text { activities for } \\
\text { occasional festivals. }\end{array}$ & $\begin{array}{c}2 \\
(0.5)\end{array}$ & $\begin{array}{c}7 \\
(1.8)\end{array}$ & $\begin{array}{c}81 \\
(21.0)\end{array}$ & $\begin{array}{c}222 \\
(57.7 \\
)\end{array}$ & $\begin{array}{c}73 \\
(19.0)\end{array}$ & 3.93 & $\begin{array}{c}0.7 \\
2\end{array}$ & High \\
\hline $\begin{array}{l}\text { 3. Intensive promotional } \\
\text { or discount activities } \\
\text { offer to market. }\end{array}$ & $\begin{array}{c}1 \\
(0.3)\end{array}$ & $\begin{array}{c}11 \\
(2.9)\end{array}$ & $\begin{array}{c}82 \\
(21.3)\end{array}$ & $\begin{array}{c}223 \\
(57.9 \\
\quad)\end{array}$ & $\begin{array}{c}68 \\
(17.7)\end{array}$ & 3.90 & $\begin{array}{c}0.7 \\
2\end{array}$ & High \\
\hline $\begin{array}{l}\text { 4. Free coupon of the } \\
\text { membership systems. }\end{array}$ & $\begin{array}{c}6 \\
(1.6)\end{array}$ & $\begin{array}{c}24 \\
(6.2)\end{array}$ & $\begin{array}{c}112 \\
(29.1)\end{array}$ & $\begin{array}{c}175 \\
(45.5 \\
)\end{array}$ & $\begin{array}{c}68 \\
(17.7)\end{array}$ & 3.71 & $\begin{array}{c}0.8 \\
8\end{array}$ & High \\
\hline \multicolumn{6}{|l|}{ Price competitiveness } & 3.50 & $\begin{array}{c}0.6 \\
2\end{array}$ & High \\
\hline $\begin{array}{l}\text { 1. Tesco Lotus offers } \\
\text { cheaper product. }\end{array}$ & $\begin{array}{c}4 \\
(1.0)\end{array}$ & $\begin{array}{l}29 \\
(7.5)\end{array}$ & $\begin{array}{c}177 \\
(46.0)\end{array}$ & $\begin{array}{c}148 \\
(38.4 \\
)\end{array}$ & $\begin{array}{l}27 \\
(7.0)\end{array}$ & 3.43 & $\begin{array}{c}0.7 \\
7\end{array}$ & High \\
\hline $\begin{array}{l}\text { 2. Clear price tags for } \\
\text { products in Tesco } \\
\text { Lotus. }\end{array}$ & $\begin{array}{c}5 \\
(1.3)\end{array}$ & $\begin{array}{c}21 \\
(5.5)\end{array}$ & $\begin{array}{c}83 \\
(21.6)\end{array}$ & $\begin{array}{c}223 \\
(57.9 \\
\quad)\end{array}$ & $\begin{array}{c}53 \\
(13.8)\end{array}$ & 3.77 & $\begin{array}{c}0.8 \\
0\end{array}$ & High \\
\hline $\begin{array}{l}\text { 3. Tesco Lotus has } \\
\text { lowest price } \\
\text { guarantee with } \\
\text { refund. }\end{array}$ & $\begin{array}{c}12 \\
(3.1)\end{array}$ & $\begin{array}{l}35 \\
(9.1)\end{array}$ & $\begin{array}{c}182 \\
(47.3)\end{array}$ & $\begin{array}{c}133 \\
(34.5 \\
)\end{array}$ & $\begin{array}{c}23 \\
(6.0)\end{array}$ & 3.31 & $\begin{array}{c}0.8 \\
4\end{array}$ & $\begin{array}{c}\text { Moderat } \\
\text { e }\end{array}$ \\
\hline \multicolumn{6}{|c|}{ Information systems service } & 3.22 & $\begin{array}{c}0.6 \\
7\end{array}$ & $\begin{array}{c}\text { Moderat } \\
\text { e }\end{array}$ \\
\hline $\begin{array}{l}\text { 1. Tesco Lotus has } \\
\text { professional product } \\
\text { advices. }\end{array}$ & $\begin{array}{c}15 \\
(3.9)\end{array}$ & $\begin{array}{c}75 \\
(19.5)\end{array}$ & $\begin{array}{c}190 \\
(49.4)\end{array}$ & $\begin{array}{c}93 \\
(24.2 \\
\quad) \\
\end{array}$ & $\begin{array}{c}12 \\
(3.1)\end{array}$ & 3.03 & $\begin{array}{c}0.8 \\
5\end{array}$ & $\begin{array}{c}\text { Moderat } \\
\mathrm{e}\end{array}$ \\
\hline
\end{tabular}


2. Tesco Lotus has provision of goods purchasing such as return or change goods.

3. Ease and convenience of use of online services.

4. Customer information retention and the initiative offers' links.

\section{Corporate image}

1. Tesco Lotus has good company's image.

2. Tesco Lotus is creditable company.

3. Tesco Lotus remains in sound financial position.

4. Tesco Lotus holds public welfare activities to the community.

$\begin{array}{cccc}6 & 34 & 172 & 141 \\ (1.6) & (8.8) & (44.7) & (36.6 \\ & & & )\end{array}$

32

(8.3)

3.41

0.8

2

High

12

(3.1)

47

$\begin{array}{cc}222 & 91 \\ (57.7) & (23.6 \\ & ) \\ 179 & 126 \\ (46.5) & (32.7 \\ & )\end{array}$

13

(3.4)

3.12

$\begin{array}{cc}0.7 & \text { Moderat } \\ 8 & \mathrm{e}\end{array}$

\section{2}

(3.1)

41

27

3.30

$\begin{array}{cc}0.8 & \text { Moderat } \\ 7 & \mathrm{e}\end{array}$

(10.6)

(7.0)

\section{$3.59 \quad 0.6 \quad$ High}

$\begin{array}{lllll}4 & 15 & 122 & 188 & 56\end{array}$

(31.7) $\quad(48.8$

(14.5)

\begin{tabular}{ll}
$3.72 \quad 0.8$ \\
\hline
\end{tabular}

High

\section{5}

13

(3.4)

$116 \quad 194$

(30.1) (50.4

57

(14.8)

$3.74 \quad 0.8$

High

5
$(1.3)$

14

(3.6)

$161 \quad 164$

41

(41.8) (42.6 (10.6)

$3.58 \quad 0.7$

0

28

\section{6}

(9.4)
116
(51.2) (30.1

\section{$3.31 \quad 0.8 \quad$ Moderat}

Regarding to table 2, it could be concluded in each construct as following detail.

Store management strategy : customers perceived this construct at high level of agreement $(\overline{\mathrm{x}}=3.78)$. Respondents prioritized Tesco Lotus has adequate products supply $(\overline{\mathrm{x}}=3.91)$, store size of Tesco Lotus is highly appropriate $(\overline{\mathrm{x}}=3.87)$, adequate chain stores close to the residential areas $(\bar{x}=3.85)$ and Tesco Lotus has sufficient employees to serve customers $(\overline{\mathrm{x}}=3.47)$ at high level respectively.

Commodity strategy : customers perceived this construct at moderate level of agreement $(\overline{\mathrm{x}}=3.38)$. They perceived Tesco Lotus has complete product range for one-stop shopping requirements $(\overline{\mathrm{x}}=3.66)$ and neatness on goods display and layout $(\overline{\mathrm{x}}=$ 3.64) at high level. In the meantime, respondents prioritized faster goods replacement when it is out of stock $(\overline{\mathrm{x}}=3.22)$, faster new product offers to the market $(\overline{\mathrm{x}}=3.21)$ and unique commodities can be found in Tesco Lotus $(\bar{x}=3.16)$ at moderate level respectively.

Promotion strategy : customers perceived this construct at high level of agreement $(\overline{\mathrm{x}}=$ 3.81). Respondents prioritized having promotional activities for occasional festivals $(\overline{\mathrm{x}}=$ $3.93)$, intensive promotional or discount activities offer to market $(\bar{x}=3.90)$, free coupon of 
the membership systems $(\overline{\mathrm{x}}=3.71)$ and Tesco Lotus offers creative promotion strategies $(\overline{\mathrm{x}}=3.69)$ at high level respectively.

Price competitiveness : customers perceived this construct at high level of agreement $(\bar{x}$ $=3.50)$. Respondents prioritized clear price tags for products in Tesco Lotus $(\overline{\mathrm{x}}=3.77)$ and Tesco Lotus offers cheaper product $(\overline{\mathrm{x}}=3.43)$ at high level respectively. In the meantime, they perceived Tesco Lotus has lowest price guarantee with refund $(\overline{\mathrm{x}}=3.31)$ at moderate level.

Information systems service : customers perceived this construct at moderate level of agreement $(\bar{x}=3.22)$. They perceived Tesco Lotus has provision of goods purchasing such as return or change goods $(\bar{x}=3.41)$ at high level. In the meantime, they also prioritized customer information retention and the initiative offers' links $(\bar{x}=3.30)$, ease and convenience of use of online services $(\bar{x}=3.12)$, and Tesco Lotus has professional product advices $(\overline{\mathrm{x}}=3.03)$ at moderate level respectively.

Corporate image : customers perceived this construct at high level of agreement $(\overline{\mathrm{x}}=$ 3.59). Respondents prioritized Tesco Lotus is creditable company $(\bar{x}=3.74)$, Tesco Lotus has good company's image $(\bar{x}=3.72)$ and Tesco Lotus remains in sound financial position $(\overline{\mathrm{x}}=3.58)$ at high level respectively. In the meantime, they perceived Tesco Lotus holds public welfare activities to the community $(\bar{x}=3.31)$ at moderate level.

\section{Part 3 : Customer Loyalty analysis}

Six questions of customer loyalty were analyzed by frequency, percent, mean, standard deviation and level of agreement. 
Table 3 Frequency, percent, mean, standard deviation and level of agreement of Customer's loyalty

$$
(n=385)
$$

\begin{tabular}{|c|c|c|c|c|c|c|c|c|}
\hline \multirow[b]{2}{*}{$\begin{array}{c}\text { Customer } \\
\text { Loyalty }\end{array}$} & \multicolumn{5}{|c|}{ Frequency (Percent) } & \multirow[b]{2}{*}{$\begin{array}{c}\text { Mea } \\
\mathbf{n}\end{array}$} & \multirow[b]{2}{*}{ SD } & \multirow[b]{2}{*}{$\begin{array}{l}\text { Level of } \\
\text { Agreem } \\
\text { ent }\end{array}$} \\
\hline & $\begin{array}{l}\text { Strong } \\
\text { ly } \\
\text { Disagr } \\
\text { ee }\end{array}$ & $\begin{array}{c}\text { Disagr } \\
\text { ee }\end{array}$ & $\begin{array}{l}\text { Neutr } \\
\text { al }\end{array}$ & $\begin{array}{c}\text { Agre } \\
\mathrm{e}\end{array}$ & $\begin{array}{c}\text { Strong } \\
\text { ly } \\
\text { Agree }\end{array}$ & & & \\
\hline Behavior & & & & & & 3.35 & $\begin{array}{c}0.6 \\
9\end{array}$ & $\begin{array}{c}\text { Moderat } \\
\mathrm{e}\end{array}$ \\
\hline $\begin{array}{l}\text { 1. You say } \\
\text { positive things } \\
\text { about Tesco } \\
\text { Lotus to } \\
\text { others. }\end{array}$ & $\begin{array}{c}6 \\
(1.6)\end{array}$ & $\begin{array}{c}36 \\
(9.4)\end{array}$ & $\begin{array}{c}212 \\
(55.1)\end{array}$ & $\begin{array}{c}114 \\
(29.6 \\
)\end{array}$ & $\begin{array}{c}17 \\
(4.4)\end{array}$ & 3.26 & $\begin{array}{c}0.7 \\
5\end{array}$ & $\begin{array}{c}\text { Moderat } \\
\mathrm{e}\end{array}$ \\
\hline $\begin{array}{l}\text { 2. You would } \\
\text { recommend } \\
\text { Tesco Lotus to } \\
\text { someone } \\
\text { seeking advice } \\
\text { on purchasing } \\
\text { goods. }\end{array}$ & $\begin{array}{c}8 \\
(2.1)\end{array}$ & $\begin{array}{c}23 \\
(6.0)\end{array}$ & $\begin{array}{c}181 \\
(47.0)\end{array}$ & $\begin{array}{c}151 \\
(39.2 \\
)\end{array}$ & $\begin{array}{c}22 \\
(5.7)\end{array}$ & 3.41 & $\begin{array}{c}0.7 \\
8\end{array}$ & High \\
\hline $\begin{array}{l}\text { 3. You } \\
\text { encourage } \\
\text { relatives and } \\
\text { friends to shop } \\
\text { at Tesco } \\
\text { Lotus. }\end{array}$ & $\begin{array}{c}9 \\
(2.3)\end{array}$ & $\begin{array}{c}20 \\
(5.2)\end{array}$ & $\begin{array}{c}198 \\
(51.4)\end{array}$ & $\begin{array}{c}132 \\
(34.3 \\
)\end{array}$ & $\begin{array}{c}26 \\
(6.8)\end{array}$ & 3.38 & $\begin{array}{c}0.7 \\
9\end{array}$ & $\begin{array}{c}\text { Moderat } \\
\mathrm{e}\end{array}$ \\
\hline Attitude & & & & & & 2.71 & $\begin{array}{c}0.6 \\
8\end{array}$ & $\begin{array}{c}\text { Moderat } \\
\mathbf{e}\end{array}$ \\
\hline $\begin{array}{l}\text { 4. You would } \\
\text { consider } \\
\text { Tesco Lotus } \\
\text { as your first } \\
\text { choice in next } \\
\text { time shopping. }\end{array}$ & $\begin{array}{c}17 \\
(4.4)\end{array}$ & $\begin{array}{c}41 \\
(10.6)\end{array}$ & $\begin{array}{c}176 \\
(45.7)\end{array}$ & $\begin{array}{c}119 \\
(30.9 \\
)\end{array}$ & $\begin{array}{c}32 \\
(8.3)\end{array}$ & 3.28 & $\begin{array}{c}0.9 \\
2\end{array}$ & $\begin{array}{c}\text { Moderat } \\
\mathrm{e}\end{array}$ \\
\hline $\begin{array}{l}\text { 5. You will not } \\
\text { patronize other } \\
\text { retailers that } \\
\text { offer more } \\
\text { attractive } \\
\text { prices. }\end{array}$ & $\begin{array}{c}74 \\
(19.2)\end{array}$ & $\begin{array}{c}111 \\
(28.8)\end{array}$ & $\begin{array}{c}133 \\
(34.5)\end{array}$ & $\begin{array}{c}55 \\
(14.3 \\
)\end{array}$ & $\begin{array}{c}12 \\
(3.1)\end{array}$ & 2.53 & $\begin{array}{l}1.0 \\
5\end{array}$ & Low \\
\hline
\end{tabular}




\section{You will switch $\quad \begin{array}{llllllll}58 & 184 & 114 & 24 & 5 & 2.31 & 0.8 & \text { Low }\end{array}$ to other retailers when $(15.1)$ $(47.8)$ (29.6) 5 there are problems with Tesco Lotus's service.}

With reference to table 3 , it could be summarized customer loyalty in two dimensions as below.

Behavior : customers perceived this construct at moderate level of agreement $(\bar{x}=3.35)$. Respondents perceived recommendation of Tesco Lotus to someone seeking advice on purchasing goods $(\overline{\mathrm{x}}=3.41)$ at high level. In the meantime, they prioritized encouraging relatives and friends to shop at Tesco Lotus $(\overline{\mathrm{x}}=3.38)$ and saying positive things about Tesco Lotus to others $(\overline{\mathrm{x}}=3.26)$ at moderate level respectively.

Attitude : customers perceived this construct at moderate level of agreement $(\bar{x}=2.71)$. Respondents perceived consideration of Tesco Lotus as their first choice in next time shopping $(\overline{\mathrm{x}}=3.28)$ at moderate level. In the meantime, they prioritized not patronizing other retailers that offer more attractive prices $(\bar{x}=2.53)$ and switching to other retailers when there are problems with Tesco Lotus's service $(\bar{x}=2.31)$ at moderate level respectively.

\section{Part 4 : What are factors attract customer to Shop Tesco-Lotus again}

Multiple regression analysis was conducted to find the answer of hypothesis testing. Multiple regressions identify the relative contribution of each variable and determine the best predictor variables among a set of variables.

Table 4 Results of Multiple Regression of hypothesis 1

\begin{tabular}{lccccc}
\hline & $\begin{array}{c}\text { Sum of } \\
\text { Squares }\end{array}$ & df & $\begin{array}{c}\text { Mean } \\
\text { Square }\end{array}$ & F & Sig. \\
\hline Regression & 90.989 & 6 & 15.165 & 62.507 & 0.000 \\
Residual & 91.706 & 378 & 0.243 & & \\
Total & 182.694 & 384 & & & \\
\hline
\end{tabular}


It showed that there was a significant linear relationship between key success factors and customer loyalty in behavior dimension with significant level at 0.05 . It could be implied that key success factors affected customer loyalty in behavior dimension.

Table 5 Multiple Regression Analysis of hypothesis 1

\begin{tabular}{|c|c|c|c|c|c|}
\hline Variable & B & SE & Beta & $\mathbf{T}$ & Sig. \\
\hline Constant & 0.302 & 0.190 & & 1.589 & 0.113 \\
\hline $\begin{array}{l}\text { Store management } \\
\text { strategy }\end{array}$ & -0.008 & 0.055 & -0.007 & -0.150 & 0.881 \\
\hline $\begin{array}{l}\text { Commodity strategy } \\
\text { Promotion strategy } \\
\text { Price competitiveness }\end{array}$ & $\begin{array}{l}0.172 \\
0.068 \\
0.066\end{array}$ & $\begin{array}{l}0.059 \\
0.053 \\
0.057\end{array}$ & $\begin{array}{l}0.156 \\
0.066 \\
0.060\end{array}$ & $\begin{array}{l}2.931 \\
1.290 \\
1.169\end{array}$ & $\begin{array}{l}0.004 \\
0.198 \\
0.243\end{array}$ \\
\hline $\begin{array}{l}\text { Information systems } \\
\text { service }\end{array}$ & 0.136 & 0.055 & 0.131 & 2.451 & 0.015 \\
\hline Corporate image & $\begin{array}{r}0.438 \\
R= \\
\text { djusted }\end{array}$ & $\begin{array}{l}0.050 \\
706 \\
=0.490 \\
\end{array}$ & 0.432 & $\begin{array}{r}8.736 \\
R^{2}=0.4 \mathrm{~S} \\
E=0.4 \mathrm{~S}\end{array}$ & 0.000 \\
\hline
\end{tabular}

As shown in table 5, key success factors (independent variable) and behavior dimension (dependent variable) had direct relationship at 0.706 . Key success factors were able to predict customer loyalty in behavior dimension $49.8 \%$. Six constructs of key success factors could be described as below.

Store management strategy : The significant of store management strategy was 0.881 which was higher than the significant standard level 0.05 , therefore store management strategy had no influence toward behavior loyalty.

Commodity strategy : The significant of commodity strategy strategy was 0.004 which was lower than the significant standard level 0.05 , therefore store commodity strategy could influence behavior loyalty, and $\mathrm{H}_{1}$ test was "Accept". Its coefficient was positive $(0.172)$ which meant both variables had direct relationship. It also indicated that there was a 0.172 increase in behavior loyalty with a unit increase in commodity strategy if other variables were fixed.

Promotion strategy : The significant of promotion strategy was 0.198 which was higher than the significant standard level 0.05 , therefore promotion strategy had no influence toward behavior loyalty. 
Price competitiveness : The significant of price competitiveness was 0.243 which was higher than the significant standard level 0.05 , therefore price competitiveness had no influence toward behavior loyalty.

Information systems service : The significant of information systems service was 0.015 which was lower than the significant standard level 0.05 , therefore information systems service could influence behavior loyalty, and $\mathrm{H}_{1}$ test was "Accept". Its coefficient was positive $(0.136)$ which meant both variables had direct relationship. It indicated that there was a 0.136 increase in behavior loyalty with a unit increase in information systems service if other variables were fixed.

Corporate image : The significant of corporate image was 0.000 which was lower than the significant standard level 0.05 , therefore corporate image could influence behavior loyalty, and $\mathrm{H}_{1}$ test was "Accept". Its coefficient was positive $(0.438)$ which meant both variables had direct relationship. It indicated that there was a 0.438 increase in behavior loyalty with a unit increase in corporate image if other variables were fixed.

Table 6 Results of Multiple Regression of hypothesis 2

\begin{tabular}{lccccc}
\hline & $\begin{array}{c}\text { Sum of } \\
\text { Squares }\end{array}$ & df & $\begin{array}{c}\text { Mean } \\
\text { Square }\end{array}$ & F & Sig. \\
\hline Regression & 51.977 & 6 & 8.663 & 26.093 & 0.000 \\
Residual & 125.497 & 378 & 0.332 & & \\
Total & 177.474 & 384 & & & \\
\hline
\end{tabular}

It showed that there was a significant linear relationship between key success factors and customer loyalty in attitude dimension with significant level at 0.05 . It could be implied that key success factors affected customer loyalty in attitude dimension. 
Table 7 Multiple Regression Analysis of hypothesis 2

\begin{tabular}{lccccc}
\hline \multicolumn{1}{c}{ Variable } & B & SE & Beta & T & Sig. \\
\hline Constant & 0.624 & 0.222 & & 2.811 & 0.005 \\
Store management & -0.126 & 0.065 & -0.107 & -1.951 & 0.052 \\
strategy & 0.263 & 0.069 & 0.243 & 3.833 & 0.000 \\
Commodity strategy & -0.023 & 0.062 & -0.022 & -0.364 & 0.716 \\
Promotion strategy & 0.113 & 0.066 & 0.103 & 1.703 & 0.089 \\
Price competitiveness & 0.108 & 0.065 & 0.106 & 1.667 & 0.096 \\
Information systems & 0.283 & 0.059 & 0.284 & 4.833 & 0.000 \\
service & $\mathrm{R}=0.541$ & & $\mathrm{R}^{2}=0.293$ \\
Corporate image & Adjusted $\mathrm{R}^{2}=0.282$ & & $\mathrm{SE}=0.576$ \\
\hline \multicolumn{5}{c}{} \\
\hline \multicolumn{5}{c}{}
\end{tabular}

As shown in table 7 , key success factors (independent variable) and attitude dimension (dependent variable) had direct relationship at 0.541 . Key success factors were able to predict customer loyalty in behavior dimension $29.3 \%$. Six constructs of key success factors could be described as below.

Store management strategy : The significant of store management strategy was 0.052 which was higher than the significant standard level 0.05 , therefore store management strategy had no influence toward behavior loyalty.

Commodity strategy : The significant of commodity strategy strategy was 0.000 which was lower than the significant standard level 0.05 , therefore store commodity strategy could influence behavior loyalty, and $\mathrm{H}_{1}$ test was "Accept". Its coefficient was positive (0.263) which meant both variables had direct relationship. It indicated that there was a 0.263 increase in attitude loyalty with a unit increase in commodity strategy if other variables were fixed.

Promotion strategy : The significant of promotion strategy was 0.716 which was higher than the significant standard level 0.05 , therefore promotion strategy had no influence toward behavior loyalty.

Price competitiveness : The significant of price competitiveness was 0.089 which was higher than the significant standard level 0.05 , therefore price competitiveness had no influence toward behavior loyalty.

Information systems service : The significant of information systems service was 0.096 which was higher than the significant standard level 0.05 , therefore information systems service had no influence toward behavior loyalty. 
Corporate image : The significant of corporate image was 0.000 which was lower than the significant standard level 0.05 , therefore corporate image could influence behavior loyalty, and $\mathrm{H}_{1}$ test was "Accept". Its coefficient was positive $(0.283)$ which meant both variables had direct relationship. It indicated that there was a 0.283 increase in attitude loyalty with a unit increase in corporate image if other variables were fixed.

Six constructs of factors attract customer to Shop Tesco-Lotus again were evaluated in frequency, percent, mean, standard deviation and level of agreement.

\section{Recommendations}

Tesco Lotus could apply below strategy to improve and develop its performance through key success factors which was ranked from highest to lowest level of agreement in customers' perspective.

1. Promotion strategy: Most customers perceived Tesco Lotus had high promotional activities for occasional festivals and intensive promotional or discount activities offer to market which lead to success. Tesco Lotus should hold promotional activities for different kind of goods each week to attract customers for visiting Tesco Lotus more often.

Free coupon can also grab attention from customers which can be at the bottom of the receipt, on the brochure, newspaper or even beside the price tag on the shelf. Hence, Tesco Lotus should enhance these kinds of activities and communicate to all target customers via various kinds of channel such as television, radio, website, newspaper, line, social media, etc.

2. Store management strategy: Most customers perceived success factors of Tesco Lotus were adequate products supply and appropriateness of store size. Therefore, Tesco Lotus should have a good inventory management so as to provide sufficient products to customers. Furthermore, if Tesco Lotus opens new branch, location should be closed to residential areas and community due to convenience transportation.

3. Corporate image: Tesco Lotus can develop its image by supporting social responsibility activity especially to be consistent to Government policy which is creating balance between conservation and sustainable utilization. For instance, Tesco Lotus can help the society reducing the use of plastic bag by offering cash refund to customers who refuse it. Since customers are quite sensitive for monetary refund rather than membership point collection. In addition, Tesco Lotus should replace plastic bag with paper bag to decrease environmental impact. 
Tesco Lotus has had various kinds of social activity for a long time such as afforestation, scholarship for Thai youth, buying fruit and vegetable directly from growers but the most important thing is all of these activities should broadcast via mass media to distribute positive attitude to all stakeholders. Tesco Lotus can also tell these stories via television in the store which customers can see and hear it all the shopping time.

4. Price competitiveness: According to the result, clear price tags and cheaper product can affect success of Tesco Lotus. Hence, Tesco Lotus has to ensure that the right price tags are appeared with the right products. On the price tags should also have enough detail like product name, product size and price which can be seen clearly and easily. On the other hand, cheaper product can induce customers to shop at Tesco Lotus because customers are price-sensitive and they have many choices to compare.

5. Commodity strategy: Tesco Lotus should offer complete product range to customers for their alternative selection which they can find what they want. Therefore, one-stop shopping store can satisfy customers' requirement and also reduce time and energy of customers to own the products.

Moreover, goods display and layout should be neat and clean because they have direct impact to customers' impression. Good perception will lead customers to spend more time in the store and can increase sales volume.

6. Information systems service: Provision of goods purchasing should be noticeable for customers. Term and condition may appear at the back of the receipt or at customer service/information counter.

If Tesco Lotus would like its customers to distribute positive communication, recommendation and encouraging to others, it should develop corporate image, commodity strategy and information systems service respectively. On the other hand, if Tesco Lotus would like to emphasize its brand in customers' mind, it should strengthen corporate image and commodity strategy respectively.

\section{Future Research}

1. In this study, it focused on only Bangkok customers because of high density of population. Nevertheless, it might not be able to represent all kind of Tesco Lotus's customers, customers who lived in other provinces should also be studied as different perception might be found. 
2. This study explored key success factors in customer perspective which was external view, therefore, further study should find out internal point of view such as management team, employee of Tesco Lotus.

3. This study applied 385 samples which may be too small. Thus, number of sample size should be increased in further study in order to gain more validity and reliability.

\section{References}

Bangkok Post. 2015. Tesco Lotus vows to keep faith in Thailand (Online). www.bangkokpost.com/archive/tesco-lotus-vows-to-keep-faith-in-thailand/ 689020., November 18, 2015.

Baran, R. J., Strunk, D. P., \& Galka, R. J. (2008). Principles of Customer Relationship Management. Ohio: Thomson South-Western.

BELLINI, S., CARDINALI, M. G. and ZILIANI, C. 2011. Building customer loyalty in retailing: not all levers are created equal. The International Review of Retail, Distribution and Consumer Research, 21(5): 461481.

Bolton, R. N., Kannan, P. K., \& Bramlett, M. (2000). Implications for Loyalty Program Membership and Service Experiences for Customer Retention and Value. Journal of Academy of Marketing Science, (28/28), 95- 108.

Bowen, J. T., \& Chen, S.-L. (2001). The Relationship between Customer Loyalty and Customer Satisfaction. International Journal of Contemporary Hospitality Management, (13), 213-217. Broekhuizen, I., \& Peelen, E. (1997). Het belonen van trouwe klanten. Tijdschrift voor Marketing, JulyAugust, 52-56.

JANOUCH, V. 2010. Internetový marketing: Prosad'te se na webu a sociálních sítích. Brno: Computer Press.

Keh, H. T., \& Lee, Y. H. (2006), Do Reward Programs Build Loyalty for Services? The Moderating Effect of Satisfaction on Type and Timing or Rewards, Journal of Retailing, 82(2), 127-136.

MRKOSOVÁ, K., DUFEK, O. and MAJER, L. 2011. Loyalty programs as part of company's marketing strategy. Acta Universitatis Agriculturae et Silviculturae Mendelianae Brunensis, LIX(2): $199-204$.

Mulhern, T. \& Duffy, D. (2004), Building Loyalty at Things Remembered, The Journal of Consumer Marketing, 21(1), 62-66. 
O'Brien, L. and C. Jones, 1995. Do Rewards Really Create Loyalty? Harvard Business Review (May-June), 75-82

OTT, B. 2011. Making Loyalty Programs Work. [Online]. Available at: http://businessjournal.gallup.com/content/149570/Making-Loyalty-ProgramsWork.aspx?version=print.

Peelen, E. (2005). Customer Relationship Management. Harlow: Pearson Education Limited. LAL, R. and BELL, D. E. 2003. The Impact of Frequent Shopper Programs in Grocery Retailing. Quantitative Marketing and Economics, 1(2): 179-202.

Raman, P. (1999). Way to Create Loyalty. New Straits Times, 17 August, Kuala Lumpur.

Reichheld, F. F., \& Sasser, W. E. (1990). Zero defections: Quality Comes to Services. Harvard Business Review, 11-105.

Reichheld, F. F., \& Teal, T. (1996). The Loyalty Effect. Harvard Business School Press. Boston, MA.

Rowley, J. (2005). Building Brand Webs: Customer Relationship Management Through the Tesco Clubcard Loyalty Scheme, International Journal of Retail \& Distribution Management, 33(3), 194-206.

VESEL, P. and ZABKAR, V. 2009. Managing custom er loyalty through the mediating role of satisfaction in the DIY retail loyalty program. Journal of Retailing and Consumer Services, 16(5): 396-406.

ZAMAZALOVÁ, M. 2009. Marketing obchodní firmy. Praha: Grada Publishing. 